\title{
Trait compassion is associated with the neural substrate of empathy
}

\author{
Xin Hou ${ }^{1,2} \cdot$ Timothy A. Allen ${ }^{3}$ - Dongtao Wei ${ }^{1,2} \cdot$ Hui Huang ${ }^{1,2} \cdot$ Kangcheng Wang ${ }^{1,2}$. \\ Colin G. DeYoung ${ }^{4}$. Jiang Qiu ${ }^{1,2}$
}

Published online: 30 June 2017

(C) Psychonomic Society, Inc. 2017

\begin{abstract}
Individual differences in the personality trait Agreeableness underlie humans' ability to interpret social cues and coordinate effectively with others. However, previous investigations of the neural basis of Agreeableness have yielded largely inconsistent results. Recent evidence has demonstrated that Agreeableness can be divided into two, correlated subdimensions. Compassion reflects tendencies toward empathy, sympathy, and concern for others, while Politeness reflects tendencies toward compliance and refraining from aggression and exploitation. The present study seeks to clarify the neural substrates of Agreeableness by examining whether structural differences in the brain show distinct associations with Compassion and Politeness. Results of a meta-analysis of fMRI studies examining empathy were used to generate hypotheses about the brain regions and networks that underlie trait Compassion. Results of a large-scale structural neuroimaging investigation $(N=275)$ were largely consistent with the meta-analysis: Compassion was positively correlated with
\end{abstract}

Xin Hou, Timothy A. Allen and Dongtao Wei contributed equally to this work.

Colin G. DeYoung

cdeyoung@umn.edu

Jiang Qiu

qiuj318@swu.edu.cn

1 Key Laboratory of Cognition and Personality (SWU), Ministry of Education, Chongqing 400715, China

2 Department of Psychology, Southwest University, Beibei, Chongqing 400715, China

3 Centre for Addiction and Mental Health, Campbell Family Mental Health Research Institute, 250 College Street, Toronto, ON M5T 1R8, Canada

4 Department of Psychology, University of Minnesota, 75 East River Rd., Minneapolis, MN 55455, USA gray matter volume in the bilateral anterior cingulate cortex (ACC) and anterior insula (AI). Further, these differences appear to be associated with Compassion specifically, as opposed to Politeness, suggesting that these two traits have at least partially distinct neuroanatomical substrates.

Keywords Compassion · Empathy $\cdot$ Personality . Neuroimaging

Human beings are inherently social animals whose survival depends on the ability to coordinate their own goals, strategies, and interpretations with those of others (DeYoung, 2015; Van Egeren, 2009). However, not all humans are equally adept in this regard; individuals vary in both their ability and their motivation to process social cues, understand the perspective of others, and work cooperatively within the social milieu. Together, individual differences in these capacities comprise the Big Five personality trait known as Agreeableness (Costa \& McRae, 1992; Goldberg, 1990; John, Naumann, \& Soto, 2008).

Personality psychologists already know a fair amount about both the nature and consequences of Agreeableness. Both lexical and questionnaire assessments of personality identify Agreeableness as one of the five broad dimensions of personality, known as the Big Five, that account for most covariation in patterns of human motivation, behavior, emotion, and cognition, as assessed by personality ratings (e.g., Goldberg, 1990; Markon, Krueger, \& Watson, 2005). The Big Five (which include Extraversion, Neuroticism, Conscientiousness, and Openness/Intellect as well as Agreeableness) are important for a wide variety of outcomes, including academic and occupational achievement, relationship success, and both physical and mental health (Ozer \& Benet-Martinez, 2006). Agreeableness specifically has been 
empirically linked to prejudice (negatively) and helping behavior (Graziano \& Habashi, 2010), conflict response and resolution (Jensen-Campbell \& Graziano, 2001), the suppression of aggressive impulses (Meier, Robinson, \& Wilkowski, 2006), and theory of mind (Nettle \& Liddle, 2008).

Although the Big Five are important, they are not the only level at which individual differences in personality are manifest. Rather, traits are arranged hierarchically, with those near the top of the hierarchy reflecting covariation among a broad range of behaviors and experiences and those near the bottom of the hierarchy reflecting more narrow and differentiated behaviors (Costa \& McCrae, 1995; Jang, McCrae, Angleitner, Riemann, \& Livesley, 1998; Markon et al., 2005). For example, traits such as altruism, empathy, compliance, modesty, honesty, and trustworthiness can all be considered markers of Agreeableness, though each also contains specific variance that is unrelated to the broader Agreeableness domain and reflects the fact that people with similar levels of general Agreeableness may nonetheless vary in the more specific traits through which they express it (Graziano \& Tobin, 2016).

Until relatively recently, most research has considered only two levels of the personality hierarchy: the Big Five domains and the narrower traits that they subsume, which have been dubbed "facets." Both factor-analytic and behavioral genetic research, however, now point to an intermediate level of traits, called aspects, existing between the Big Five and their respective facets (DeYoung, Quilty, \& Peterson, 2007). In a behavior genetic analysis of twins, Jang and colleagues found that two genetic factors were necessary to account for the covariation among facets measuring each of the Big Five domains in the NEO-PI-R (a popular measure of the Big Five; Jang, Livesley, Angleitner, Riemann, \& Vernon, 2002). DeYoung and colleagues (2007) later replicated this finding in a nongenetic factor analysis using 15 facet scales for each domain, showing that, once again, covariation among facets was best accounted for by two correlated factors per domain. The 10 resulting factors were further characterized by examining their correlation with more than 2,000 items of the International Personality Item Pool (Goldberg, 1999), leading to the creation of the Big Five Aspect Scales (BFAS), a 100-item self-report questionnaire assessing each of the 10 aspects that comprise the Big Five. The 10 aspect-level traits are particularly useful because they represent an empirically derived substructure for the Big Five and are likely to represent the most important distinctions for discriminant validity within each Big Five domain. In contrast, there is no consensus regarding the number and identity of the narrower, facet-level traits in each domain (e.g., Costa \& McCrae, 1992; Goldberg, 1999).

Within Agreeableness, the two aspects were labeled Compassion and Politeness (DeYoung et al., 2007). Compassion reflects the capacity to care about others emotionally, including tendencies toward empathy, sympathy, and interpersonal concern, whereas Politeness reflects a motivation to conform to social norms and resist aggressive, exploitative, or socially disruptive impulses. Empirical research on Compassion and Politeness has demonstrated the predictive utility of the two aspects, above and beyond any effects explained by Agreeableness more broadly. For instance, the two aspects are differentially related to political ideology and moral values, prosocial decision making, and various manifestations of psychopathology (DeYoung, Carey, Krueger, \& Ross, 2016; Hirsh, DeYoung, Xu, \& Peterson, 2010; Zhao, Ferguson, \& Smillie, 2016).

Research investigating the role of Agreeableness and its aspects in predicting important life outcomes is steadily growing (for a review, see Graziano \& Tobin, 2013). However, very little is currently known about the underlying psychological and biological mechanisms that explain why individuals differ on these traits. The lack of a coherent explanatory framework underlying the Big Five has been acknowledged by many in recent years, leading to increased efforts to identify the causal mechanisms underlying trait variation (e.g., Denissen \& Penke, 2008; Nettle, 2006, 2007; Van Egeren, 2009). In the present study, we adopt the most thoroughly elaborated of these theoretical foundations, Cybernetic Big Five Theory (CB5T), in order to formulate hypotheses about the psychological and biological systems that may underlie variation in Agreeableness and its aspects (Allen \& DeYoung, 2017; DeYoung, 2015).

Cybernetics is the study of goal-directed, self-regulating systems (Carver \& Scheier, 1998; Wiener, 1961). CB5T posits that personality traits result from variation in the parameters of evolved cybernetic mechanisms that allow people to identify and obtain their goals. Regarding the biological instantiation of these mechanisms, CB5T adopts the MIMIC (multiple indicators, multiple causes) approach (cf. Kievit et al., 2012), which specifies that a shared psychological function causes covariance among the narrower traits (the multiple indicators) that are encompassed by each of the Big Five or their aspects, but that this psychological function is instantiated by complex brain systems with many parameters (the multiple causes). Hence, CB5T does not attempt to identify just a single biological parameter responsible for a given trait but rather takes a systems approach because it recognizes that multiple biological mechanisms contribute to any given psychological function.

Unlike Extraversion and Neuroticism, which have been extensively studied in personality neuroscience because of their links to basic reward and threat processes and their inclusion in older personality models (for reviews, see Allen \& DeYoung, 2017; Shackman, Tromp, Stockbridge, Kaplan, Tillman, \& Fox 2016; Wacker \& Smillie, 2015), Agreeableness has received relatively little attention. Two small resting state fMRI studies found that Agreeableness is positively associated with functional connectivity within major hubs of the default network (Adelstein et al., 2011; 
Sampaio et al., 2014), a finding consistent with the theory that understanding and anticipating the mental states of others is facilitated by this network (Andrews-Hanna, Smallwood, \& Spreng, 2014). Evidence from structural MRI studies, however, is mixed, with some studies finding no associations (Bjørnebekk et al., 2013; Liu et al., 2013) and others finding inconsistent associations (DeYoung et al., 2010; Hu et al., 2011; Kapogiannis, Sutin, Davatzikos, Costa, \& Resnick, 2013). We suspect that one way to clarify the association of Agreeableness with brain structure will be to examine its two aspects separately.

Here, we focus on Compassion because of its link to empathy, which has been defined as the ability to understand and share in another's emotional state (Singer \& Klimecki, 2014). Questionnaire-based studies of personality show that empathy is positively associated with variation in Agreeableness, but more specifically with Compassion (DeYoung et al., 2007; DeYoung et al., 2016). We are referring here to trait Compassion, as measured by the BFAS and other similar personality measures (for example, one item from the BFAS Compassion scale is, "I feel others' emotions"; DeYoung et al., 2007). Trait Compassion is a broad construct that reflects tendencies toward interpersonal concern, empathy, and prosocial behavior. This stands in some contrast to the more narrow compassion construct frequently employed within social-cognitive theories of empathy, which refers specifically to tendencies to show concern and care for another (but not necessarily empathy with another; see Singer \& Klimecki, 2014, for an overview). For the remainder of this article, note that we are referring specifically to trait Compassion whenever we use the term Compassion.

Empathy is a multifaceted construct, which includes affective, cognitive, and regulatory components that govern the generation of shared emotional experience, the recognition or understanding that one's emotions match those of another, and the top-down regulation of the empathic response, respectively (Decety, 2011; Decety \& Jackson, 2004). Neurobiological research has shown that each of these components may have at least partially distinct neural substrates (Decety \& Jackson, 2004). Two regions that appear to be particularly central to empathy are the anterior insula (AI) and the anterior cingulate cortex (ACC), which have been shown to be active both during the firsthand experience of pain and while observing others who are in pain (Fan, Duncan, de Greck, \& Northoff, 2011; Lamm, Decety, \& Singer, 2011; Peyron, Laurent, \& Garcia-Larrea, 2000). These two regions demonstrate increased activation during the vicarious observation of other affective states as well (Fan et al., 2011; for a review, see Bernhardt \& Singer, 2012). Importantly however, the AI and ACC are not the only brain regions of relevance to empathy. Rather, empathy is likely to draw on an expansive network of regions, including limbic areas governing the generation of emotion, and prefrontal regions involved in the regulation of emotional experience and the facilitation of adaptive behavioral responses (Decety, 2011).

Given that variations in empathy are central to Compassion, we can use the extensive MRI literature on empathy to gain traction. Compared to studies of general Agreeableness, there have been a larger number of studies of trait differences in empathy, and a much larger number of studies of empathy as a process or state (not focused on individual differences). As a result, research documenting the neural basis of empathy may prove instructive for identifying brain regions involved in the Compassion aspect of Agreeableness. Notably, the exact nature of relations between brain structure and brain function remains unclear (DeYoung et al., 2010). However, there is clear evidence that patterns of brain functioning have an important role in shaping the brain's structural architecture (e.g., Boyke, Driemeyer, Gaser, Büchel, \& May, 2008), which suggests it is reasonable to use the functional correlates of a phenomenon to generate hypotheses regarding its structural correlates as well. We therefore tested the hypothesis that Compassion would be associated with brain structure in regions overlapping with those implicated in empathy through meta-analysis of the fMRI literature. We also tested whether this association was specific to Compassion or also shared with Politeness, the other aspect of Agreeableness.

\section{Materials and method}

\section{Preliminary meta-analysis of fMRI studies on empathy}

In order to identify regions and networks of the brain implicated in empathic processing, we first performed a metaanalysis of extant fMRI studies of empathy. Neurosynth (Yarkoni, Poldrack, Nichols, Van Essen, \& Wager, 2011), an online meta-analysis tool, was used to perform the meta-analysis. Neurosynth combines text-mining, meta-analysis, and machine-learning techniques in order to sift through previously published empirical studies and generate probabilistic mappings of regions in the brain that are activated in the context of specific cognitive, affective, or motivational variables that are of interest to the researcher. Researchers can select keywords and then harness the meta-analytic capabilities of Neurosynth in order to quantify the relations between brain activation and the construct of interest. We selected empathy as our keyword because, as mentioned previously, it is closely related to the trait of Compassion, and Neurosynth does not yet contain an active database of studies explicitly focused on Compassion. Images were thresholded in order to correct for multiple comparisons using a false discovery rate (FDR) of $p<.01$. Using the search term empathy, Neurosynth identified a database of 137 studies. Results of the present study focus on forward 
inference activation maps generated by Neurosynth; these maps quantify changes in brain activity given a known psychological manipulation, allowing the researcher to therefore make a forward inference about the neural substrates primarily associated with a given state.

\section{Main analysis: participants}

Healthy, right-handed participants $(N=275 ; 155$ females $)$ were recruited from an undergraduate population attending Southwest University in Chongqing, China, to participate in an ongoing neuroimaging project investigating associations between personality and neurobiological variables $\left(M_{\text {age }}=\right.$ $19.92, S D=1.31$ ). With a sample of 275 , we have $80 \%$ power to detect effects equivalent to a correlation of .17, thus we are reasonably well powered to detect the range of typical effects found both in research on individual differences specifically and in psychology generally (Gignac \& Szodorai, 2016; Hemphill, 2003).

The experimental protocol for this was approved by the Southwest University Brain Imaging Center Institutional Review Board. All participants provided written, informed consent and were compensated for their participation. The Structured Clinical Interview for DSM-IV (First, Spitzer, Gibbon, \& Williams, 2002) was administered to all participants in order to screen for any past or present psychiatric disorders. Any participants reporting past or present neurological conditions, use of psychotropic medications (or other medications that could influence brain function), loss of consciousness, or head trauma were also excluded. Participants were selected for the present study if they had completed the BFAS and had complete neuroimaging data.

\section{Personality assessment}

Personality was assessed using the BFAS (DeYoung et al., 2007), a 100-item self-rating questionnaire measuring each of the Big Five domains and the 10 lower order aspects (two aspects comprise each domain). Participants are asked to rate the extent to which they agree with BFAS items on a 5-point scale, ranging from $1=$ strongly disagree to $5=$ strongly agree. All BFAS items were translated into Chinese for the purposes of this study. In the present analysis, we focused on the two aspect scales that make up the broader Agreeableness domain: Compassion (e.g., "Feel others' emotions") and Politeness (e.g., "Respect authority").

\section{MRI data acquisition}

A 3-Tesla Siemens Trio MRI scanner (Siemens Medical, Erlangen, Germany) was used to acquire a whole-brain magnetization-prepared rapid gradient-echo image (MPRAGE) for each subject. One three-dimensional high-resolution T1- weighted image was acquired from each subject using the following parameters: repetition time $=1,900 \mathrm{~ms}$; echo time $=2.52 \mathrm{~ms}$; inversion time $=900 \mathrm{~ms}$; flip angle $=9^{\circ}$; field of view $=256 \times 256 \mathrm{~mm}^{2}$; number of slices $=176$; slice thickness $=1.0 \mathrm{~mm}$; voxels size $=1 \times 1 \times 1 \mathrm{~mm}^{3}$.

\section{Preprocessing of structural data}

Structural images were processed using the Statistical Parametric Mapping software (SPM8; Wellcome Trust Centre for Neuroimaging, University College London, UK; http://www.fil.ion.ucl.ac.uk/spm) executed in MATLAB 7.8 (MathWorks Inc., Natick, MA, USA).

To remove gross anatomical abnormalities, all of the structural images were first displayed in SPM8. Images were manually reoriented and fixed to the anterior commissure for more accurate registration. Subsequently, the new segmentation option in SPM8 was used to segment the structural images into six tissues: white matter, gray matter, cerebrospinal fluid, skull, soft tissue outside the brain, air, and other material outside the head. The DARTEL algorithm (diffeomorphic anatomical registration through exponentiated lie algebra; Ashburner, 2007) was used for registration, normalization, and modulation. DARTEL registration involves first computing the specific template using the average tissue probability maps from all participants, and then warping each participant's segmented maps into the specific template. This procedure is carried out repeatedly in order to improve alignment and achieve more accurate intersubject registration, eventually yielding the optimal study-specific template. To ensure conservation of regional differences in the absolute amounts of gray matter, the image intensity of each voxel was modulated by Jacobian determinants. Registered images were then transformed to Montreal Neurological Institute (MNI) space. Finally, images were smoothed with a $10-\mathrm{mm}$ full-width half-maximum Gaussian filter to enhance the signal-to-noise ratio.

\section{Statistical analyses}

Statistical analyses were performed using SPM8. In order to test our main hypothesis, brain regions identified by their association with empathy in our Neurosynth meta-analysis were combined into a single anatomical mask, and a small volume correction was performed to account for multiple comparisons. Multiple linear regressions controlling for age, sex, and global gray matter volume (GMV) were used to determine whether Compassion and Politeness were significantly associated with GMV in brain regions included in the aforementioned mask. On an exploratory basis, we were also interested in identifying any regions of the brain that were outside the anatomical mask derived from our empathy meta-analysis but nonetheless still associated with trait Compassion. To examine 
this question, multiple linear regressions were used in wholebrain analyses to identify regions where regional GMV was associated with individual differences in personality. Participant scores on the aspect of interest (Compassion or Politeness) were entered as the predictor variable. Participant age, sex, and global GMV were entered as covariates in order to control for potential confounds. To avoid edge effects around the borders between gray matter and white matter, an absolute threshold masking of . 2 was used; that is, voxels with gray matter values lower than .2 were excluded from analyses (Mühlau et al., 2005). In all analyses, the clusterlevel statistical threshold was set at $p<.05$, and corrected using FWE cluster correction, with an initial voxel-level threshold of $p<.005$.

\section{Results}

\section{Meta-analytic findings}

Forward inference activation maps from the Neurosynth metaanalysis of empathy fMRI studies are presented in Fig. 1. Results of the meta-analysis indicate that fMRI studies that induce or manipulate empathy consistently report activation in the bilateral anterior insula (AI), the anterior cingulate cortex (ACC), amygdala, and parahippocampal gyrus (thresholded at $p<.01$, FDR-corrected; see Table 1).

\section{Main analysis}

The mean Compassion score for the current sample was 3.72 $(S D=.43)$, while the mean Politeness score was $3.34(S D=$ .39). No significant gender differences were found for scores on Compassion $(t=.53, p=.62)$. Females scored significantly higher on Politeness than did males $(t=2.04, p=.04)$. As anticipated, Compassion was significantly positively associated with Politeness $(r=.14, p=.02)$.

In order to test our main hypothesis that trait Compassion is associated with regions of the brain that are also linked to empathy, we performed a small volume correction using a single anatomical mask defined by all regions identified in the aforementioned Neurosynth meta-analysis, including bilateral AI, ACC, amygdala, and parahippocampal gyrus. Multiple regressions controlling for age, sex, and global GMV showed that BFAS Compassion was positively and significantly associated with GMV in two regions included within the mask: the ACC $(r=.21)$ and the bilateral AI (left: $r=$ .26; right: $r=.20$; see Table 2 and Fig. 2). (Correlations are with mean cluster values, controlling for all covariates from the regression.) No significant negative correlations were found between Compassion scores and GMV. Further, no associations between Politeness and GMV were significant following the small volume correction. Follow-up tests indicated that the correlations with Compassion were significantly greater than the correlations with Politeness in all three regions: ACC, $t(272)=2.18, p=.03$; left AI, $t(272)=2.95, p$ $=.003$; and right AI, $t(272)=2.53, p=.01$ (see Fig. 3).

\section{Whole-brain analysis}

In a secondary, more exploratory analysis, designed to detect regions that might have been missed by constraining our primary analysis to the mask, we examined whether any regions in the whole brain were associated with trait Compassion or Politeness. Multiple linear regressions were used in wholebrain analyses to identify areas in which regional GMV was significantly associated with either trait. Controlling for age, sex, and global GM volumes, trait Compassion was positively and significantly associated with GMV in the bilateral ACC $(r$ $=.23$; see Table 3 ). Sizeable clusters were also detected at the voxel level in bilateral insula as well as amygdala/ parahippocampal gyrus and precuneus, but they were not large enough to survive the FWE correction. Follow-up tests indicated that the strength of the correlation between Compassion and the ACC was significantly different from the strength of the correlation between Politeness and the ACC, $t(272)=2.50, p=.01$. No significant negative correlations were found between Compassion scores and GMV, and no significant associations of any kind were found between Politeness and regional GMV, although there was a large cluster that passed the voxel threshold in middle occipital cortex.

\section{Discussion}

The present study investigated whether regional gray matter volumes are differentially related to Compassion and Politeness, two aspect-level traits encompassed by the broader Big Five domain of Agreeableness. Specifically, we hypothesized that associations between GMV and trait Compassion would largely overlap with regions identified in a metaanalysis of the fMRI literature on empathy. Our data provide clear evidence that brain regions underlying empathic processing are likewise linked to individual differences in trait Compassion. Moreover, these findings did not extend to trait Politeness, suggesting that the two aspects of Agreeableness are associated with distinct neuroanatomical substrates and reinforcing the utility of studying the aspect-level traits.

Trait Compassion was positively associated with GMV in the bilateral ACC and AI within a large sample of healthy volunteers. These findings are partially consistent with the results of our meta-analysis on fMRI studies examining empathy, which found that empathic processing is associated with activation in the bilateral ACC, AI, amygdala, and parahippocampal gyrus. On an exploratory basis, we also conducted whole brain analyses to examine whether any 


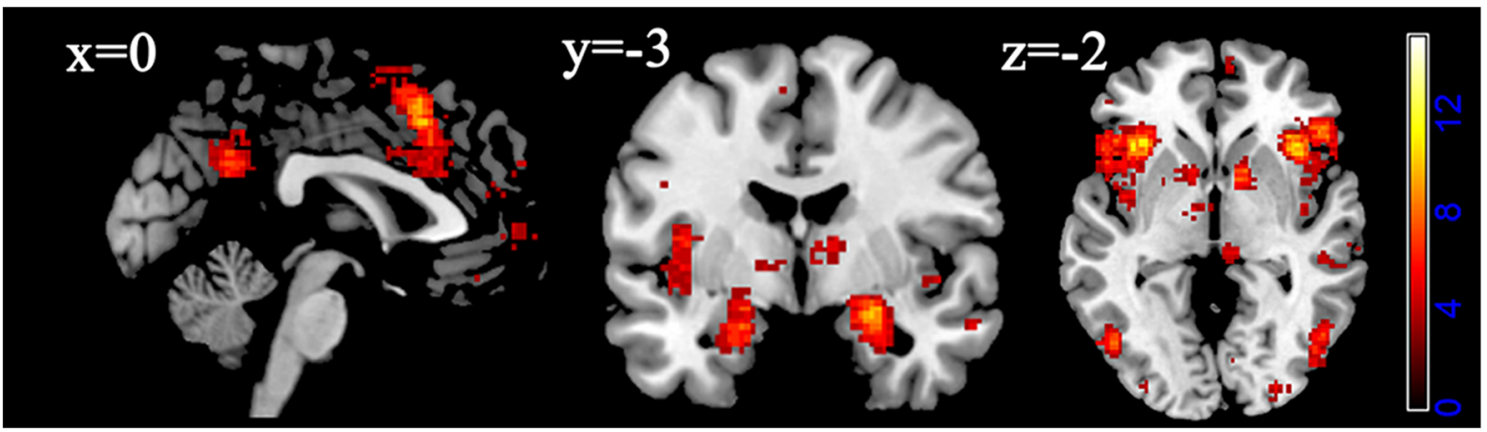

Fig. 1 Results from Neurosynth meta-analysis of fMRI studies on empathy. Displays the meta-analytic forward-inference map generated from the search term empathy, thresholded at $p<.01$, FDR-corrected. The color bar represents the $t$ value. (Color figure online)

additional brain regions might be associated with either Compassion or Politeness. In this analysis, only the bilateral ACC was significantly associated with trait Compassion. However, this appeared to be due to lack of power, stemming from the need to correct for multiple tests across the whole brain. Although AI was not significantly associated with Compassion in the whole-brain analysis, large clusters in bilateral AI crossed the voxel-level threshold (consistent with our main analysis), as did smaller but still substantial clusters in amygdala/parahippocampal gyrus and precuneus. Crossing the voxel-level threshold indicates that these effects were of similar magnitude to those we detected in our main analysis, even though their spatial extent was not large enough to survive FWE correction. This exploratory analysis suggests that the true associations of trait Compassion with GMV may be even more extensive in regions linked to empathy than our study was able to show. Future studies with larger samples could have the statistical power necessary to identify the full extent of brain regions associated with Compassion. Trait Politeness was unrelated to volume in any of the regions linked to Compassion, reinforcing the conclusion that our main findings are specific to Compassion and are not a function of the broader Agreeableness domain.
Previous research indicates that both the AI and the ACC are central to understanding and sharing in another's pain. In a recent meta-analysis of fMRI studies on empathy for pain, Lamm and colleagues (2011) found that both regions are active when experiencing pain personally and when observing the pain of others, supporting the notion that the brain may use personal experience to access and understand the experience of others. Importantly, this network appears to be active during empathy for social and emotional forms of pain as well (as opposed to just physical pain), as another meta-analysis of empathy studies likewise found consistent activation of the ACC and AI across different types of stimulus categories (Fan et al., 2011).

The results of our own meta-analysis indicate that functional activation of both the ACC and AI is associated with empathy generally, as studies of both state and trait empathy were included in the meta-analysis. Whereas state empathy involves a momentary experience that is elicited when observing and sharing in the emotional or affective state of another (e.g., seeing someone stub a toe and sharing in that pain), trait empathy reflects a relatively stable inclination to understand and share in the affective state of others across contexts. Singer and colleagues (2004) found that trait empathy, as

Table 1 Brain regions associated with empathy in Neurosynth meta-analysis

\begin{tabular}{|c|c|c|c|c|c|c|}
\hline \multirow[t]{2}{*}{ Region } & & \multicolumn{3}{|c|}{ MNI coordination } & \multirow[t]{2}{*}{ Cluster size (voxels) } & \multirow{2}{*}{$\begin{array}{l}\text { Peak } \\
t \text { value }\end{array}$} \\
\hline & & $x$ & $y$ & $z$ & & \\
\hline \multirow[t]{2}{*}{ Insula } & $\mathrm{L}$ & -34 & 22 & 0 & 2158 & $15.02 *$ \\
\hline & $\mathrm{R}$ & 38 & 22 & -8 & 2063 & $12.16^{*}$ \\
\hline ACC/Medial frontal gyrus & $\mathrm{L} / \mathrm{R}$ & -6 & 20 & 42 & 1511 & $12.16^{*}$ \\
\hline Precuneus & $\mathrm{L} / \mathrm{R}$ & -6 & -54 & 30 & 310 & $8.59 *$ \\
\hline Amygdala/parahippocampal gyrus & $\mathrm{R}$ & 26 & -6 & -14 & 512 & $10.73 *$ \\
\hline \multirow[t]{2}{*}{ Postcentral gyrus } & $\mathrm{L}$ & -58 & -24 & 38 & 327 & $10.02 *$ \\
\hline & $\mathrm{R}$ & 62 & -22 & 38 & 341 & $10.02 *$ \\
\hline \multirow[t]{2}{*}{ Middle temporal gyrus } & $\mathrm{L}$ & -48 & -68 & 0 & 679 & $10.02 *$ \\
\hline & $\mathrm{R}$ & 50 & -66 & -2 & 773 & $7.16^{*}$ \\
\hline
\end{tabular}

$* p<.01($ FDR corrected) 
Table 2 Brain regions associated with trait compassion following small volume correction.

\begin{tabular}{|c|c|c|c|c|c|c|c|}
\hline \multirow[t]{2}{*}{ Brain regions } & & \multicolumn{3}{|c|}{ MNI coordination } & \multirow[t]{2}{*}{ Cluster size (voxels) } & \multirow{2}{*}{$\begin{array}{l}\text { Peak } \\
t \text { value }\end{array}$} & \multirow{2}{*}{$\begin{array}{l}\text { Adjusted } \\
p \text { value }\end{array}$} \\
\hline & & $x$ & $y$ & $z$ & & & \\
\hline \multirow[t]{2}{*}{ AI } & $\mathrm{L}$ & -33 & 0 & 14 & 594 & 4.40 & $.028 *$ \\
\hline & $\mathrm{R}$ & 45 & 9 & 6 & 246 & 3.31 & $.034 *$ \\
\hline $\mathrm{ACC}$ & $\mathrm{L} / \mathrm{R}$ & -2 & 24 & 29 & 431 & 3.55 & $.034 *$ \\
\hline
\end{tabular}

$* p<.05$ (small volume corrected)

measured by the Empathic Concerns subscale of the Interpersonal Reaction Index and the Balanced Emotional Empathy Scale, was associated with increased activation in the bilateral ACC and left AI, consistent with our own results. In a much larger sample, Bernhardt and colleagues found that empathy was positively related to increased connectivity of the AI with regions of the prefrontal cortex and limbic system, regardless of whether empathy was measured as a state (during tasks) or as a trait, using self-report questionnaires (Bernhardt, Klimecki, Leiberg, \& Singer, 2013). Reinforcing the importance of the insula, a recent study found that, compared to healthy controls and patients with noninsular gliomas, patients with insular gliomas scored lower on trait empathy and performed worse on several tasks measuring recognition of facial emotion, perspective-taking, and pain perception (Chen et al., 2016). Relatedly, several large structural MRI studies have found that empathy is positively associated with volume in the AI (Eres, Decety, Louis, \& Molenberghs, 2015; Mutschler, Reinbold, Wankerl, Seifritz, \& Ball, 2013; Sassa et al., 2012) and the ACC (Banissy, Kanai, Walsh, \& Rees, 2012; Eres et al., 2015). Other studies in both humans and animals have found that the link between empathy and the ACC is likely to be confined to the gyral, as opposed to the sulcal, portion of the ACC (for reviews, see Apps, Rushworth, \& Chang, 2016; Lockwood, 2016). This is consistent with our own findings, which show the link between trait Compassion and the ACC appears to be centralized the gyral portion of the ACC. The gyrus of the ACC is densely connected to a variety of brain regions implicated in social cognition and mentalizing (Lockwood, 2016), which may partially explain previous links between Agreeableness and theory of mind (Nettle \& Liddle, 2008). Taken together with the results of the Neurosynth meta-analysis reported here, this literature suggests that the same neural substrates underlie state and trait empathy, and that trait empathy and Compassion are overlapping constructs with shared neurobiological underpinnings (Allen \& DeYoung, 2017; DeYoung et al., 2007; 2016). Empathy may be the major psychological mechanism that causes people to be compassionate.

Previous studies examining the neuroanatomical substrates of agreeableness have yielded either null (Bjørnebekk et al., 2013; Liu et al., 2013) or inconsistent findings (DeYoung et al., 2010; Hu et al., 2011; Kapogiannis et al., 2013). The results of our study suggest that one way to avoid these inconsistencies may be to examine the two aspects of agreeableness separately. This method would be consistent with CB5T, which suggests that, although Compassion and Politeness may both covary due to a shared psychological function (coordination of goals within the social milieu), they should nonetheless have at least partially distinct neural substrates (Allen \& DeYoung, 2017). Our results are consistent with this hypothesis in that Politeness was unrelated to regions implicated in Compassion. Less research relevant to the neurobiological foundations of Politeness has been conducted, in

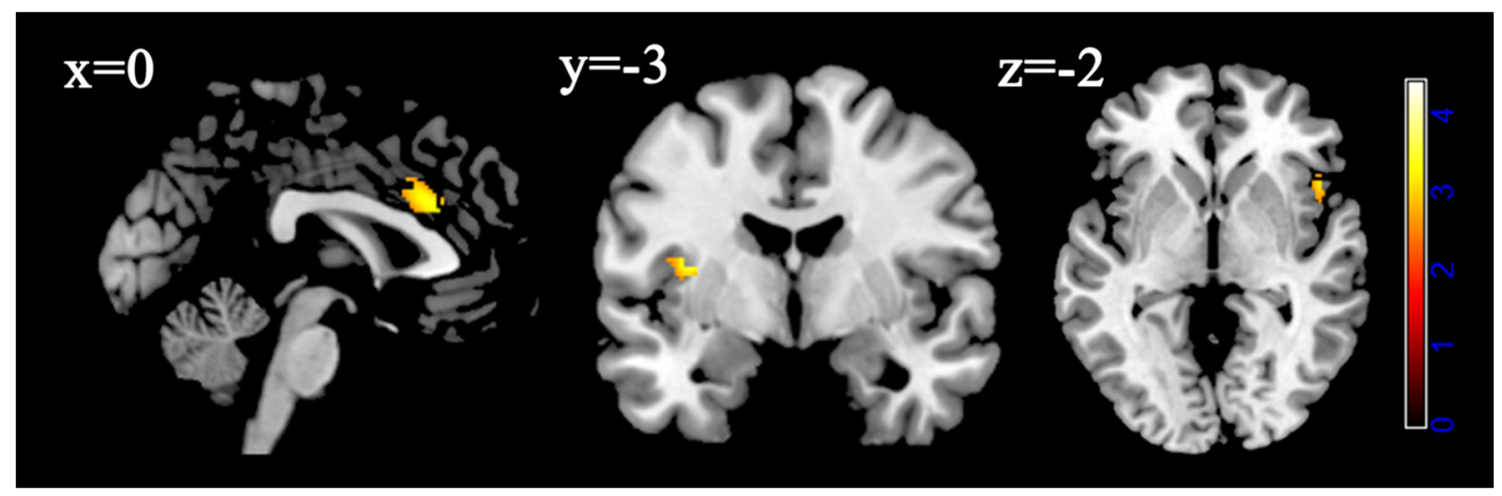

Fig. 2 Results of a small volume correction using all significant regions from the Neurosynth meta-analysis as a mask, $p<.05$. The color bar represents the $t$ value. (Color figure online) 

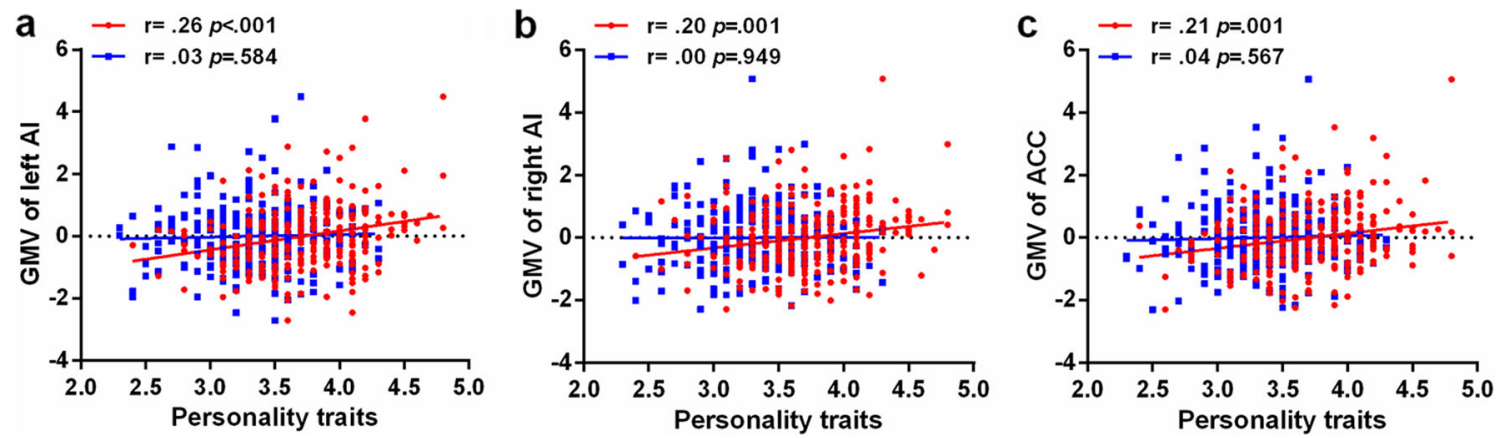

Fig. 3 Correlations between the two Agreeableness aspects and regions of the brain that were significantly associated with trait Compassion following small volume correction $(\mathrm{red}=$ trait Compassion; blue $=$ trait Politeness). (Color figure online)

comparison with all the research on empathy that is relevant to trait Compassion, and future work should attempt to develop hypotheses regarding the neural substrates of Politeness.

When interpreting our findings it is important consider that the relation between brain structure and brain function remains unclear. The finding that trait Compassion is linked to increased volume of the bilateral AI and ACC generally coincides with functional studies showing increased activation in these regions as well (Singer et al., 2004), which would suggest that volume and function may be positively related in this context. Nonetheless, it is most prudent simply to interpret the present findings as implicating the AI and ACC in the neurobiological substrate of Compassion without drawing strong conclusions about their implications for brain function. Additional research will be necessary to determine how
Compassion relates to the functional activation of these regions.

In conclusion, the findings from the present study indicate that trait Compassion and empathic processing share neuroanatomical substrates. In contrast, the Politeness aspect of the broader Big Five Agreeableness domain is not associated with the same structural correlates. Consistent with the overarching goal of CB5T, these findings represent one step toward integrating the biological and psychological mechanisms that produce individual differences in trait Compassion.

Acknowledgments This research was supported by the National Natural Science Foundation of China (31271087; 31470981; 31571137; 31500885), National Outstanding Young People Plan, the Program for the Top Young Talents by Chongqing, the Fundamental Research Funds

Table 3 Brain regions associated with Compassion and Politeness in exploratory whole-brain analysis

\begin{tabular}{|c|c|c|c|c|c|c|c|}
\hline \multirow[t]{2}{*}{ Brain regions } & & \multicolumn{3}{|c|}{ MNI coordination } & \multirow[t]{2}{*}{ Cluster size (voxels) } & \multirow{2}{*}{$\begin{array}{l}\text { Peak } \\
t \text { value }\end{array}$} & \multirow{2}{*}{$\begin{array}{l}\text { Adjusted } \\
p \text { value }\end{array}$} \\
\hline & & $x$ & $y$ & $z$ & & & \\
\hline \multicolumn{8}{|l|}{ Compassion } \\
\hline \multirow[t]{2}{*}{ AI } & $\mathrm{L}$ & -32 & 0 & 15 & 2076 & 4.61 & .118 \\
\hline & $\mathrm{R}$ & 31 & 6 & 14 & 1141 & 3.65 & .443 \\
\hline $\mathrm{ACC}$ & $\mathrm{L} / \mathrm{R}$ & 0 & 37 & 21 & 2888 & 4.00 & $.039 *$ \\
\hline \multirow[t]{2}{*}{ Parahippocampal gyrus/amygdala } & $\mathrm{L}$ & -15 & -20 & -28 & 511 & 3.50 & .884 \\
\hline & $\mathrm{R}$ & 18 & -17 & -28 & 235 & 3.15 & .989 \\
\hline Precuneus & $\mathrm{R}$ & 9 & -77 & 32 & 429 & 3.17 & .929 \\
\hline \multirow[t]{2}{*}{ Superior frontal } & $\mathrm{L}$ & -16 & -2 & 62 & 67 & 2.77 & 1.00 \\
\hline & $\mathrm{R}$ & 12 & -14 & 53 & 96 & 3.50 & .999 \\
\hline Cerebellum & $\mathrm{L} / \mathrm{R}$ & -2 & -62 & 1 & 136 & 2.95 & .998 \\
\hline \multicolumn{8}{|l|}{ Politeness } \\
\hline \multirow[t]{2}{*}{ Middle occipital } & $\mathrm{L}$ & -22 & -96 & 23 & 97 & 2.81 & .998 \\
\hline & $\mathrm{R}$ & 39 & -84 & 18 & 2624 & 4.47 & .131 \\
\hline IFG & $\mathrm{L}$ & -48 & 30 & 18 & 272 & 3.42 & .979 \\
\hline OFC & $\mathrm{L} / \mathrm{R}$ & -3 & 28 & -25 & 335 & 3.28 & .965 \\
\hline
\end{tabular}

Note. $\mathrm{GMV}=$ gray matter volume; $\mathrm{AI}=$ anterior insula; $\mathrm{ACC}=$ anterior cingulate cortex; $\mathrm{IFG}=$ inferior frontal gyrus; $\mathrm{OFC}=$ orbitofrontal cortex. $* p<$ .05 (FWE corrected) 
for the Central Universities (SWU1509383, SWU1509451), Natural Science Foundation of Chongqing (cstc2015jcyjA10106), Fok Ying Tung Education Foundation (151023), General Financial Grant from the China Postdoctoral Science Foundation (2015M572423, 2015M580767), Special Funds from the Chongqing Postdoctoral Science Foundation (Xm2015037), Key research for humanities and social sciences of the Ministry of Education (14JJD880009).

\section{Compliance with ethical standards}

Conflicts of interest The authors declare no conflicts of interest.

\section{References}

Adelstein, J. S., Shehzad, Z., Mennes, M., DeYoung, C. G., Zuo, X.-N., Kelly, C., . . . Milham, M. P. (2011). Personality is reflected in the brain's intrinsic functional architecture. PLOS ONE, 6, e27633. doi: 10.1371/journal.pone. 0027633

Allen, T., \& DeYoung, C. (2017). Personality neuroscience and the five factor model. In T. A. Widiger (Ed.), Oxford handbook of the five factor model. New York, NY: Oxford University Press.

Andrews-Hanna, J., Smallwood, J., \& Spreng, R. N. (2014). The default network and self-generated thought: Component processes, dynamic control, and clinical relevance. Annals of the New York Academy of Sciences, 1316, 29-52. doi:10.1111/nyas. 12360

Apps, M. A., Rushworth, M. F., \& Chang, S. W. (2016). The anterior cingulate gyrus and social cognition: Tracking the motivation of others. Neuron, 90(4), 692-707. doi:10.1016/j.neuron.2016.04.018

Ashburner, J. (2007). A fast diffeomorphic image registration algorithm. NeuroImage, 38, 95-113. doi:10.1016/j.neuroimage.2007.07.007

Banissy, M. J., Kanai, R., Walsh, V., \& Rees, G. (2012). Inter-individual differences in empathy are reflected in human brain structure. NeuroImage, 62(3), 2034-2039. doi:10.1016/j.neuroimage.2012. 05.081

Bernhardt, B. C., Klimecki, O. M., Leiberg, S., \& Singer, T. (2013). Structural covariance networks of the dorsal anterior insula predict females' individual differences in empathic responding. Cerebral Cortex, 24, 2189-2198. doi:10.1093/cercor/bht072

Bernhardt, B. C., \& Singer, T. (2012). The neural basis of empathy. Annual Review of Neuroscience, 35, 1-23. doi:10.1146/annurevneuro-062111-150536

Bjørnebekk, A., Fjell, A. M., Walhovd, K. B., Grydeland, H., Torgersen, S., \& Westlye, L. T. (2013). Neuronal correlates of the five factor model (FFM) of human personality: Multimodal imaging in a large healthy sample. NeuroImage, 65, 194-208. doi:10.1016/j. neuroimage.2012.10.009

Boyke, J., Driemeyer, J., Gaser, C., Büchel, C., \& May, A. (2008). Training-induced brain structure changes in the elderly. Journal of Neuroscience, 28(28), 7031-7035. doi:10.1523/JNEUROSCI. 0742-08.2008

Carver, C., \& Scheier, M. (1998). On the self-regulation of behavior. New York, NY: Cambridge University Press.

Chen, P., Wang, G., Ma, R., Jing, F., Zhang, Y., Wang, Y., . . Zhang, X. (2016). Multidimensional assessment of empathic abilities in patients with insular glioma. Cognitive, Affective, \& Behavioral Neuroscience, 1-14. doi:10.3758/s13415-016-0445-0

Costa, P. T., Jr., \& McCrae, R. R. (1992). NEO PI-R professional manual. Odessa, FL: Psychological Assessment Resources.

Costa, P. T., Jr., \& McCrae, R. R. (1995). Domains and facets: Hierarchical personality assessment using the Revised NEO Personality Inventory. Journal of Personality Assessment, 64, 2150. doi:10.1207/s15327752jpa6401_2
Decety, J. (2011). Dissecting the neural mechanisms mediating empathy. Emotion Review, 3(1), 92-108. doi:10.1177/1754073910374662

Decety, J., \& Jackson, P. L. (2004). The functional architecture of human empathy. Behavioral and Cognitive Neuroscience Reviews, 3(2), 71-100. doi:10.1177/1534582304267187

Denissen, J., \& Penke, L. (2008). Motivational individual reaction norms underlying the five-factor model of personality: First steps towards a theory-based conceptual framework. Journal of Research in Personality, 42, 1285-1302. doi:10.1016/j.jrp.2008.04.002

DeYoung, C. G. (2015). Cybernetic big five theory. Journal of Research in Personality, 56, 33-58. doi:10.1016/j.jrp.2014.07.004

DeYoung, C. G., Carey, B., Krueger, R., \& Ross, S. (2016). Ten aspects of the Big Five in the personality inventory for DSM-5. Personality Disorders: Theory, Research, and Treatment, 7, 113-123. doi:10. 1037/per0000170

DeYoung, C. G., Hirsh, J. B., Shane, M. S., Papademetris, X., Rajeevan, N., \& Gray, J. R. (2010). Testing predictions from personality neuroscience: Brain structure and the Big Five. Psychological Science, 21, 820-828. doi:10.1177/0956797610370159

DeYoung, C. G., Quilty, L. C., \& Peterson, J. B. (2007). Between facets and domains: 10 aspects of the Big Five. Journal of Personality and Social Psychology, 93, 880-896. doi:10.1037/0022-3514.93.5.880

Eres, R., Decety, J., Louis, W. R., \& Molenberghs, P. (2015). Individual differences in local gray matter density are associated with differences in affective and cognitive empathy. NeuroImage, 117, 305310. doi:10.1016/j.neuroimage.2015.05.038

Fan, Y., Duncan, N. W., de Greck, M., \& Northoff, G. (2011). Is there a core neural network in empathy? An fMRI based quantitative metaanalysis. Neuroscience \& Biobehavioral Reviews, 35, 903-911. doi: 10.1016/j.neubiorev.2010.10.009

First, M. B., Spitzer, R. L., Gibbon, M., \& Williams, J. B. (2002). Structured clinical interview for DSM-IV-TR Axis I disorders (Research version patientth ed.). New York, NY: Biometrics Research, New York State Psychiatric Institute.

Gignac, G. E., \& Szodorai, E. T. (2016). Effect size guidelines for individual differences researchers. Personality and Individual Differences, 102, 74-78. doi:10.1016/j.paid.2016.06.069

Goldberg, L. R. (1990). An alternative "description of personality": The Big-Five factor structure. Journal of Personality and Social Psychology, 59(6), 1216-1229.

Goldberg, L. (1999). A broad-bandwidth, public domain, personality inventory measuring the lower-level facets of several five-factor models. In I. Mervielde, I. J. Deary, F. De Fruyt, \& F. Ostendorf (Eds.), Personality psychology in Europe (Vol. 7, pp. 7-28). Tilburg, Netherlands: Tilburg University Press.

Graziano, W. G., \& Habashi, M. M. (2010). Motivational processes underlying both prejudice and helping. Personality and Social Psychology Review, 14, 313-331. doi:10.1177/1088868310361239

Graziano, W. G., \& Tobin, R. M. (2013). The cognitive and motivational foundations underlying agreeableness. In M. D. Robinson, E. R. Watkins, \& E. Harmon-Jones (Eds.), Handbook of cognition and emotion (pp. 347-364). New York, NY: Guilford Press.

Graziano, W. G., \& Tobin, R. M. (2016). Agreeableness and the five factor model. In T. A. Widiger (Ed.), Oxford handbook of the five factor model. New York, NY: Oxford University Press.

Hemphill, J. F. (2003). Interpreting the magnitudes of correlation coefficients. American Psychologist, 58, 78-79. doi:10.1037/0003-066X. 58.1 .78

Hirsh, J. B., DeYoung, C. G., Xu, X., \& Peterson, J. B. (2010). Compassionate liberals and polite conservatives: Associations of agreeableness with political ideology and moral values. Personality and Social Psychology Bulletin, 36, 655-664. doi:10. 1177/0146167210366854

Hu, X., Erb, M., Ackermann, H., Martin, J. A., Grodd, W., \& Reiterer, S. M. (2011). Voxel-based morphometry studies of personality: Issue of statistical model specification-Effect of nuisance covariates. 
NeuroImage, 54, 1994-2005. doi:10.1016/j.neuroimage.2010.10. 024

Jang, K. L., Livesley, W. J., Angleitner, A., Riemann, R., \& Vernon, P. A. (2002). Genetic and environmental influences on the covariance of facets defining the domains of the five-factor model of personality. Personality and Individual Differences, 33, 83-101. doi:10.1016/ S0191-8869(01)00137-4

Jang, K. L., McCrae, R. R., Angleitner, A., Riemann, R., \& Livesley, W. J. (1998). Heritability of facet-level traits in a cross-cultural twin sample: Support for a hierarchical model of personality. Journal of Personality, 74, 1556-1565. doi:10.1037/0022-3514.74.6.1556

Jensen-Campbell, L. A., \& Graziano, W. G. (2001). Agreeableness as a moderator of interpersonal conflict. Journal of Personality, 69, 323 362. doi:10.1111/1467-6494.00148

John, O., Naumann, L., \& Soto, C. (2008). Paradigm shift to the integrative big five trait taxonomy. In O. P. John, R. W. Robins, \& L. A. Pervin (Eds.), Handbook of personality: Theory and research (pp. 114-158). New York, NY: Guilford Press.

Kapogiannis, D., Sutin, A., Davatzikos, C., Costa, P., \& Resnick, S. (2013). The five factors of personality and regional cortical variability in the Baltimore Longitudinal Study of Aging. Human Brain Mapping, 34, 2829-2840. doi:10.1002/hbm.22108

Kievit, R. A., van Rooijen, H., Wicherts, J. M., Waldorp, L. J., Kan, K. J., Scholte, H. S., \& Borsboom, D. (2012). Intelligence and the brain: A model-based approach. Cognitive Neuroscience, 3, 89-97. doi:10. 1080/17588928.2011.628383

Lamm, C., Decety, J., \& Singer, T. (2011). Meta-analytic evidence for common and distinct neural networks associated with directly experienced pain and empathy for pain. NeuroImage, 54, 2492-2502. doi:10.1016/j.neuroimage.2010.10.014

Liu, W. Y., Weber, B., Reuter, M., Markett, S., Chu, W. C., \& Montag, C. (2013). The Big Five of personality and structural imaging revisited. NeuroReport, 24, 375-380. doi:10.1097/WNR.0b013e328360dad7

Lockwood, P. L. (2016). The anatomy of empathy: Vicarious experience and disorders of social cognition. Behavioural Brain Research, 311, 255-266. doi:10.1016/j.bbr.2016.05.048

Markon, K. E., Krueger, R. F., \& Watson, D. (2005). Delineating the structure of normal and abnormal personality: An integrative hierarchical approach. Journal of Personality and Social Psychology, 88, 139-157. doi:10.1037/0022-3514.88.1.139

Meier, B. P., Robinson, M. D., \& Wilkowski, B. M. (2006). Turning the other cheek: Agreeableness and the regulation of aggression-related primes. Psychological Science, 17, 136-142. doi:10.1111/j.14679280.2006.01676.x

Mühlau, M., Rauschecker, J. P., Oestreicher, E., Gaser, C., Röttinger, M., Wohlschläger, A. M., ... Sander, D. (2005). Structural brain changes in tinnitus. Cerebral Cortex, 16, 1283-1288. doi:10.1093/cercor/ bhj070

Mutschler, I., Reinbold, C., Wankerl, J., Seifritz, E., \& Ball, T. (2013). Structural basis of empathy and the domain general region in the anterior insular cortex. Frontiers in Human Neuroscience, 7, 1-18. doi:10.3389/fnhum.2013.00177
Nettle, D. (2006). The evolution of personality variation in humans and other animals. American Psychologist, 61, 622-631. doi:10.1037/ 0003-066X.61.6.622

Nettle, D. (2007). Personality: What makes you the way you are. New York, NY: Oxford University Press.

Nettle, D., \& Liddle, B. (2008). Agreeableness is related to social-cognitive, but not social-perceptual, theory of mind. European Journal of Personality, 22, 323-335. doi:10.1002/per.672

Ozer, D., \& Benet-Martinez, V. (2006). Personality and the prediction of consequential outcomes. Annual Review of Psychology, 57, 401421. doi:10.1146/annurev.psych.57.102904.190127

Peyron, R., Laurent, B., \& Garcia-Larrea, L. (2000). Functional imaging of brain responses to pain. A review and meta-analysis. Neurophysiologie Clinique/Clinical Neurophysiology, 30(5), 263288. doi:10.1016/S0987-7053(00)00227-6

Sampaio, A., Soares, J. M., Coutinho, J., Sousa, N., \& Gonçalves, Ó. F. (2014). The Big Five default brain: Functional evidence. Brain Structure and Function, 219, 1913-1922. doi:10.1007/s00429013-0610-y

Sassa, Y., Taki, Y., Takeuchi, H., Hashizume, H., Asano, M., Asano, K., . . . Kawashima, R. (2012). The correlation between brain gray matter volume and empathizing and systemizing quotients in healthy children. NeuroImage, 60, 2035-2041. doi:10.1016/j.neuroimage.2012. 02.021

Shackman, A. J., Tromp, D. P., Stockbridge, M. D., Kaplan, C. M., Tillman, R. M., \& Fox, A. S. (2016). Dispositional negativity: An integrative psychological and neurobiological perspective. Psychological Bulletin, 142(12), 1275-1314. doi:10.1037/ bul0000073

Singer, T., \& Klimecki, O. M. (2014). Empathy and compassion. Current Biology, 24(18), R875-R878. doi:10.1016/j.cub.2014.06.054

Singer, T., Seymour, B., O’Doherty, J., Kaube, H., Dolan, R. J., \& Frith, C. D. (2004). Empathy for pain involves the affective but not sensory components of pain. Science, 303, 1157-1162. doi:10.1126/ science. 1093535

Van Egeren, L. F. (2009). A cybernetic model of global personality traits. Personality and Social Psychology Review, 13, 92-108. doi:10. $1177 / 1088868309334860$

Wacker, J., \& Smillie, L. D. (2015). Trait extraversion and dopamine function. Social and Personality Psychology Compass, 9(6), 225238. doi:10.1111/spc3.12175

Wiener, N. (1961). Cybernetics - Or control and communication in the animal and the machine (2nd ed.). New York, NY: MIT Press/ Wiley. doi:10.1037/13140-000

Yarkoni, T., Poldrack, R. A., Nichols, T. E., Van Essen, D. C., \& Wager, T. D. (2011). Large-scale automated synthesis of human functional neuroimaging data. Nature Methods, 8, 665-670. doi:10.1038/ nmeth. 1635

Zhao, K., Ferguson, E., \& Smillie, L. D. (2016). Individual differences in good manners rather than compassion predict fair allocations of wealth in the dictator game. Journal of Personality, 1-13. doi:10. 1111/jopy. 12237 